A $R$ R T T I I C C U L L O $\begin{array}{lllllllll}R & E & F & L & E & X & I & O & N\end{array}$

\section{ANÁlisis y PROPUESTAS DE MEJORA SOBRE LA INVESTIGACIÓN EN LAS UNIVERSIDADES ECUATORIANAS ${ }^{1}$}

\author{
ANALYSIS AND PROPOSALS OF IMPROVEMENT IN RESEARCH \\ CONDUCTED IN ECUADORIAN UNIVERSITIES
}

Por: Manuel Ferrer Muñoz*

${ }^{*}$ Docente-Investigador en la Universidad Técnica del Norte (Ibarra, Ecuador). Doctor en Filosofía y Letras, Sección de Historia.

${ }^{1}$ Este artículo forma parte de un proyecto de investigación que, con el título "Las artesanías en San Antonio de Ibarra: prospectivas de modernización en los procesos productivos y de un crecimiento urbano respetuoso con el medioambiente $y$ el patrimonio histórico" cocure se Técnica del Norte (Ibarra, Ecuador)
Recibido: 25 de febrero de 2016 - Aprobado: 14 de julio de 2016

\section{RESUMEN}

El texto plantea un análisis de los logros y de las falencias de la investigación en las universidades ecuatorianas, con objeto de sustentar propuestas concretas orientadas a la mejora del trabajo investigativo que se desarrolla en esas instituciones. Se destaca, muy en particular, que las potencialidades de los investigadores se vean coartadas por un exceso de control y una preocupación desmedida por la planificación.

Palabras clave: Investigación, Universidades, Ecuador, Plan Nacional del Buen Vivir.

This text presents an analysis of the research achievements and needs analysis in the research conducted in ecuadorian universities, in order to support specific proposals pointing to improve the research work carried out in these institutions. The emphasis is particularly concentrated on the fact that the potential of researchers is being limited by an overloaded control and disproportionate preoccupation for planning processes.

Keywords: Research, Universities, Ecuador, National Plan of Good Living.

\section{Presentación}

n un reciente trabajo, que se halla en vías de publicación, hemos indagado 1 acerca de las causas por las que la investigación en las universidades ecuatorianas no acaba de remontar el vuelo, y sobre la necesidad de una profunda renovación en el modo en que se contemplan las tareas investigadoras en el seno de los centros de educación superior del Ecuador. Subrayábamos en ese escrito que la Universidad no puede configurarse como un coto de poder, ni como un refugio de mediocres, ni como un invento para disimular el desempleo juvenil. Al definir el recinto universitario como el espacio donde florecen propuestas, sensatas o incendiarias por su talante

\section{ABSTRACT}


radicalmente innovador, y donde despuntan el espíritu crítico, el afán de búsqueda, el contraste de pareceres, enfatizábamos que la investigación constituye una actividad inherente al quehacer universitario y consustancial con él, que se erige como la salvaguarda de aquellas potencialidades: sin ella, la docencia queda en simple recitación mecánica de sabiduría ajena.

Todo lo expuesto hasta aquí fundamenta la conveniencia de completar el panorama crítico que trazamos entonces con un análisis más detenido que permita sustentar propuestas concretas orientadas a la mejora del trabajo investigativo que se desarrolla en las universidades del Ecuador.

\section{Fuentes para el análisis y guías para la investigación en el Ecuador}

- as observaciones practicadas para fundamentar las propuestas que se contienen en este artículo parten de la información proporcionada por lel Consejo de Evaluación, Acreditación y Aseguramiento de la Calidad de la Educación Superior (CEAACES), dependiente de la Secretaría Nacional de Educación Superior, Ciencia, Tecnología e Innovación (Senescyt) del Gobierno del Ecuador; de varias decenas de entrevistas mantenidas con docentes de universidades de Quito y de la Zona 1 (Carchi, Esmeraldas, Imbabura y Sucumbíos), y del informe que la investigadora Ayolaida Rodríguez Miranda presentó como resultado de su estancia en dicha Zona 1 con una beca Prometeo.

El Plan Nacional del Buen Vivir (PNBV) constituye la guía por la que discurren las líneas de acción del Gobierno cada cuatro años. En el actualmente vigente (2013-2017) se establece una planificación en los diversos ámbitos, con el objeto declarado de "gobernar para profundizar el cambio" (Plan Nacional del Buen Vivir, 2013: 14) que representó la acometida de la construcción del Socialismo del Buen Vivir por el equipo de gobierno triunfante en 2007 a través de la propuesta de Revolución Ciudadana. Por medio de esa planificación se pretende que la investigación se adecue a las diversas condiciones ambientales y culturales locales y a los requerimientos del entorno, y que se oriente en gran medida a la satisfacción de las necesidades del sector productivo mediante una ágil coordinación de los entes investigadores.

La Agenda de la Zona 1, que, como las demás agendas zonales, pretende concretar espacialmente los objetivos del PNBV, subraya la importancia que adquirió la puesta en marcha de la Ciudad del Conocimiento Yachay, un centro académico con carreras técnicas en cinco áreas, desarrollado en el cantón Urcuquí de la provincia de Imbabura, que, a pesar del empuje con que nació, 
se halla enfrentado a serias dificultades que pueden llevar a comprometer su continuidad a largo plazo; incluye propuestas para guiar la investigación hacia la mejora de los recursos productivos de la región, y se compromete a "fortalecer las identidades a través de la investigación-difusión de sus expresiones culturales y artísticas" (Agenda Zonal 1, 2013: 136), con un énfasis particular en la preservación de las lenguas nativas y del patrimonio cultural tangible e intangible.

\section{Panorama de los resultados observados}

T o cierto es que, pese a esas declaraciones de buenas intenciones, y en lo que respecta a la Zona 1, a las alturas de diciembre de 2015 podía lafirmarse que, en términos generales, la investigación carecía de una estructura organizacional, y que en la mayoría de los casos no existían personas encargadas de la coordinación de las actividades de investigación. Más aún, no se disponía de financiamiento para actividades de investigación, ni se contaba con estructuras de apoyo; y tampoco existían facilidades para el acceso a la red, ni equipos de computación actualizados. Resulta llamativo que la Universidad Técnica del Norte, con todo su potencial, hubiera puesto en marcha tan solo 29 proyectos a principios de aquel año (Rodríguez Miranda, A., 2015): y no se olvide que un indicador de la planificación de la investigación del CEAACES es, precisamente, la existencia de líneas y políticas institucionales de investigación de la institución de educación superior.

El análisis de las líneas de investigación de las instituciones de la Zona 1 revela algunas evidencias interesantes acerca del impacto de los objetivos del PNBV en esas líneas de investigación y en el número de instituciones que las respaldan. Como puede apreciarse en el cuadro que se muestra más abajo, solo siete líneas de investigación apuntan al logro del objetivo $1^{\circ}$ (consolidar el Estado democrático y la construcción del poder popular), y solo nueve líneas, al objetivo $6^{\circ}$ (consolidar la transformación de la justicia y fortalecer la seguridad integral, en estricto respeto a los derechos humanos), lo cual no hace sino corroborar que, entre los estudiosos de las ciencias sociales, los dedicados al cultivo del derecho adolecen particularmente de escaso interés por las tareas investigativas. En el caso de la Zona 1, apenas algunos trabajos de tesis de la Escuela de Jurisprudencia de la Pontificia Universidad Católica del Ecuador, sede Ibarra, acometen esa temática, de particular importancia en una región donde radica un elevado número de refugiados colombianos, expulsados de su país por el conflicto de la guerrilla, que viven situaciones muy difíciles (particularmente en el caso de algunas mujeres, sometidas a explotación sexual). 
Paradójicamente, el objetivo $4^{\circ}$ del PNBV (fortalecer las capacidades y potencialidades de la ciudadanía) es el que de modo más frecuente aparece contemplado en esas líneas de investigación. Le siguen, en orden descendente, los objetivos $10^{\circ}, 3^{\circ}, 2^{\circ}, 8^{\circ} \ldots$ Cabría cuestionarse la robustez de las bases sobre las que debe asentarse en la Zona 1 el fortalecimiento de las potencialidades de la ciudadanía que preconiza el objetivo $4^{\circ}$, si el sustento jurídico e institucional de esa tarea aparece tan descuidado en los enfoques investigadores de las universidades ecuatorianas del norte.

\begin{tabular}{|c|c|c|c|}
\hline $\begin{array}{l}\text { Número de } \\
\text { objetivo del PNBV }\end{array}$ & Enunciado & $\begin{array}{c}\text { Líneas de } \\
\text { investigación que lo } \\
\text { mencionan y \% } \\
\end{array}$ & $\begin{array}{l}\text { Instituciones que } \\
\text { acogen esas líneas }\end{array}$ \\
\hline 4 & $\begin{array}{l}\text { Fortalecer las capacidades y potencialidades de } \\
\text { la ciudadanía. }\end{array}$ & $53(14,52 \%)$ & 14 \\
\hline 10 & $\begin{array}{l}\text { Impulsar la transformación de la matriz } \\
\text { productiva. }\end{array}$ & $51(13,97 \%)$ & 12 \\
\hline 3 & Mejorar la calidad de vida de la población. & $42(11,50 \%)$ & 10 \\
\hline 2 & $\begin{array}{l}\text { Auspiciar la igualdad, la cohesión, la inclusión } \\
\text { y la equidad social y territorial, en la } \\
\text { diversidad. }\end{array}$ & $37(10,13 \%)$ & 9 \\
\hline 8 & $\begin{array}{l}\text { Consolidar el sistema económico social y } \\
\text { solidario, de forma sostenible. }\end{array}$ & $35(9,58 \%)$ & 8 \\
\hline 11 & $\begin{array}{l}\text { Asegurar la soberanía y eficiencia de los } \\
\text { sectores estratégicos para la transformación } \\
\text { industrial y tecnológica. }\end{array}$ & $33(9,04 \%)$ & 8 \\
\hline 5 & $\begin{array}{l}\text { Construir espacios de encuentro común } \\
\text { y fortalecer la identidad nacional, las } \\
\text { identidades diversas, la plurinacionalidad y la } \\
\text { interculturalidad. }\end{array}$ & $32(8,76 \%)$ & 10 \\
\hline 7 & $\begin{array}{l}\text { Garantizar los derechos de la naturaleza } \\
\text { y promover la sostenibilidad ambiental } \\
\text { territorial y global. }\end{array}$ & $25(6,84 \%)$ & 10 \\
\hline 9 & $\begin{array}{l}\text { Garantizar el trabajo digno en todas sus } \\
\text { formas. }\end{array}$ & $23(6,30 \%)$ & 8 \\
\hline 12 & $\begin{array}{l}\text { Garantizar la soberanía y la paz, profundizar } \\
\text { la inserción estratégica en el mundo y la } \\
\text { integración latinoamericana. }\end{array}$ & $18(4,93 \%)$ & 4 \\
\hline 6 & $\begin{array}{l}\text { Consolidar la transformación de la justicia } \\
\text { y fortalecer la seguridad integral, en estricto } \\
\text { respeto a los derechos humanos. }\end{array}$ & $9(2,46 \%)$ & 4 \\
\hline \multirow[t]{2}{*}{1} & $\begin{array}{l}\text { Consolidar el Estado democrático y la } \\
\text { construcción del poder popular. }\end{array}$ & $7(1,91 \%)$ & 2 \\
\hline & & TOTAL: 365 (100\%) & \\
\hline
\end{tabular}

Fuente: Rodríguez Miranda, A., 2015 
${ }^{2}$ Parece que hubiera

caído en saco roto la acuciosa búsqueda emprendida en los años setenta del pasado siglo

por el Departamento de

por el Departamento

de Ciencias Human

y el Centro de Estudios

Latinoamericanos de la

Pontificia Universidad

Católica del Ecuador,

en persecución de una

filosofía latinoamerica-

na en la que pudiera in-

sertarse el pensamiento

ecuatoriano (Roig, A.
A., 2013, 183-191).
Ciertamente, la modestia de los logros observados en la investigación, tanto en la Zona 1 como en la capital de la República, se explica por el abandono en que se ha encontrado tradicionalmente esta tarea en el Ecuador. Una prueba de ello viene procurada por el bajísimo puntaje obtenido por el parámetro correspondiente a la investigación en el proceso de categorización de las universidades llevado a cabo por el CEAACES en 2013: en promedio, 0,2 sobre 1 (El Universo, 15 de diciembre de 2013). Ese organismo hubo de reconocer que, incluso en aquellas universidades categorizadas como A -el nivel más elevado-, la producción científica de alto impacto se concentraba en un reducido número de docentes investigadores, y que se hallaba por debajo de los estándares internacionales (CEAACES, 2013 b: 19).

Se detecta, además, la carencia de una política universitaria que conciba la investigación como una estrategia de desarrollo a largo plazo y que valore las actividades a ella vinculadas, en la misma medida y con el mismo interés con que se cuidan los docentes o las de vinculación, porque se desprecia todo conocimiento que carezca de aplicaciones inmediatas, y porque no existe la indispensable planificación ${ }^{2}$. En consecuencia, los profesores se ven imposibilitados para planear, desarrollar proyectos de investigación y divulgarlos a través de los eventos en que participan colegas de universidades de otros países, por la sencilla razón de que no suelen -ini pueden!- concurrir. Ni habitualmente disponen de tiempo para ello ni se les proporcionan los imprescindibles estímulos: porque apenas hay financiación para participación en congresos (sobre todo, en las universidades privadas), y porque las gestiones burocráticas retrasan excesivamente la adquisición de la seguridad de que se cuenta con recursos para asistir, por lo que, razonablemente, se opta por la inhibición antes de adquirir compromisos que no se sabe si podrán cumplirse.

La formación de muchos docentes de universidades de la Zona 1 en el ámbito de la investigación no es, ciertamente, la más adecuada (Rodríguez Miranda, A., 2015); y no resulta en absoluto aventurado extender el diagnóstico a la mayoría de las instituciones de grado superior del Ecuador. Desprovistos muchos profesores de bases para el desarrollo de la investigación y desconocedores de las metodologías aplicables en sus áreas de conocimiento, resulta poco menos que imposible que se adentren en la experiencia investigadora, sin que basten episódicos talleres de capacitación, que, habida cuenta del desconocimiento y desinterés de muchos miembros de los claustros académicos, no dejan de constituir simples paliativos. Esto sin contar con que la mayoría de los cursos de formación que se organizan están orientados a la docencia, porque 
tradicionalmente ninguna universidad ecuatoriana cimentó su prestigio en la investigación (Ruiz, J. C., 2001) y porque, como observó en su momento Felipe Burbano de Lara, muchas universidades se conciben a sí mismas como de docencia y no de investigación (El Universo, 15 de diciembre de 2013), temerosas de enfrentarse el reto que comporta impulsar la investigación -y sus correspondientes técnicas- desde la propia institución universitaria (Pérez Escobar, J., 2013: 9-11).

La escasa inclinación a investigar, asociada a una cultura del facilismo y al profundo desánimo instalado en los profesores veteranos, no podrá ser subsanada por las medios implementados actualmente desde algunos departamentos de investigación, convertidos en telarañas de procedimientos y regidos por estructuras piramidales, que dejan fuera a los que deberían ser los protagonistas de la investigación. Esos departamentos corren el riesgo de convertirse en máquinas ejecutivas, redactoras y recopiladoras de informes, transmisoras de consignas y fiscalizadoras implacables de resultados, que agobian más que orientan, que exigen más que sirven, que controlan más que estimulan, y que persiguen con más celo la satisfacción de las formalidades administrativas que los logros de objetivos en la investigación. Más se asemejan a dependencias gubernamentales, donde la jefatura reviste caracteres autoritarios, que a órganos universitarios inspirados por el gobierno colegiado, la pasión por el conocer, el amor al estudio, la inquietud intelectual, la cercanía a la sociedad civil y a las fuentes de financiación.

La misma concepción de muchos proyectos de investigación acogidos por los referidos departamentos está inspirada más en normas administrativas y contables que en la habilitación de recursos para el desarrollo de las investigaciones. Se entiende así que los criterios que inspiran los presupuestos sean escasamente operativos, que se prevean asignaciones muy cuestionables, y que la disponibilidad de los recursos liberados se produzca a destiempo. Y es que, ciertamente, no basta la presencia de Ph. Ds. para generar investigación, sino que se requiere la asignación puntual de los recursos en los tiempos marcados en los cronogramas de los proyectos, no sujeta a los caprichosos ritmos de torpes mecanismos administrativos. Ese es el contexto en que se inserta la preocupación expresada por uno de los investigadores entrevistados: "si la universidad quiere realmente actualizarse y mejorar su nivel académico y de investigación, el cambio tiene que ser real y efectivo: de lo contrario nosotros no podemos dar los resultados para los cuales hemos sido contratados". 
Los sistemas de evaluación, frecuentemente más atentos a los procedimientos que a los resultados; la continua rendición de cuentas, y los medios habilitados para la medición de los avances sirven de poca ayuda en el proceso de desarrollo de proyectos. Causan tedio entre los investigadores los exagerados trámites burocráticos, los prescindibles procesos de control y socialización y el reclamo de constataciones físicas con remisión a plazos que a menudo no guardan relación con la naturaleza de las investigaciones y que las entorpecen en numerosas ocasiones. Se da más importancia a la presentación de esas evidencias - a veces escasamente fundamentadas- que a la dotación de los recursos que necesita el investigador para llevar a cabo su proyecto en las mejores condiciones.

La limitación de recursos disponibles se relaciona muy estrechamente con la ausencia de vínculos entre las universidades y los círculos empresariales del medio en que ejercen su actividad, perceptible por la inexistencia de políticas de relaciones institucionales orientadas a lograr la captación de recursos de empresas del entorno. Se echa en falta en muchos casos una Asociación de Antiguos Alumnos, que, si consiguiera evitar la deriva burocratizante, podría significar un interesante aporte económico y servir de instrumento para la captación de alumnos.

\section{¿Qué soluciones se proponen para emplazar a la investigación en el lugar prioritario que le corresponde?}

\footnotetext{
7 iertamente, una cultura de investigación no se improvisa y no puede ignorar los ritmos pausados de procesos orgánicos, por lo que el camino hacia su conformación será largo y azaroso -aunque apasionante-, y solo posible si existen políticas institucionales que otorguen prioridad a la investigación y la conciban como "ejercicio de comprensión reflexiva y de crítica fundamentada de la sociedad" (Villavicencio, A., 2013: 6-8). Pero sí deben emprenderse iniciativas que, con amplitud de miras, apunten a ese objetivo. De ellas irá tratándose en las páginas que siguen.
}

Por supuesto, en primerísimo lugar se impone una política de estímulos a la productividad académica, a través de programas que impliquen una evaluación anual de publicaciones y de participación en congresos. De acuerdo con los resultados obtenidos en función de los baremos que se establezcan en cada caso, los profesores podrían recibir a final de año un estímulo salarial cuyo monto tendría que comprometerse y concretarse antes de la puesta en marcha de estos planes. 
Todo docente debería tener derecho a que la institución a la que se halla vinculado cubra todos los gastos que comporte su participación en un congreso anual, siempre que se trate de un evento que se desarrolle en territorio nacional. En el caso de reuniones científicas que se celebren en el extranjero habría que estudiar la viabilidad de que la universidad de adscripción asumiera un porcentaje de los costos. Y, al mismo tiempo, tendrían que proporcionarse al académico los instrumentos adecuados para el ejercicio de sus tareas de investigación: computadoras (también para los becarios), impresoras, escáneres, laboratorios, maquinaria... Lo anterior no obsta para que, con carácter general, se revise la política de salarios de las universidades, de manera que paulatinamente se logre aproximar los sueldos que se perciben en unas y otras.

Para impulsar el proceso de eliminación de obstáculos que frenan la dedicación a tareas de investigación, se recomienda descartar las convocatorias institucionales que impliquen una asistencia obligatoria. Se trata de implantar una nueva cultura de la comunicación, que facilite los procesos de inducción y de socialización, sin necesidad de estar paralizando periódicamente las actividades de toda la universidad, con daño indudable para la investigación y la docencia.

Con objeto de propiciar espacios fecundos de diálogo y de debate informal, se sugiere suprimir las restricciones existentes -donde las haya- para el acceso a las cafeterías que funcionan en las instalaciones universitarias: unas trabas causantes de profundo malestar y desconcierto entre los profesores que vienen de otras latitudes. Por el contrario, deben favorecerse encuentros entre alumnos y profesores que se enmarquen precisamente en esas áreas de descanso (Fernández Pérez, R.; Gámiz Ruiz, M. A.; García Martínez, M. A.; Moraga Ávila, J.; Peña Sánchez, J., y Porras Mínguez, S., 2012: 129-130).

Hay que tratar de propiciar que los docentes-investigadores se sientan a gusto en las instituciones que los acogen, de modo que dejen de producirse continuas vacantes y sucesivos relevos. Para ello, además del programa de estímulos mencionado en párrafo anterior, se sugiere un plan articulado, que constaría de los elementos que se enumeran a continuación.

Se precisa una revisión a fondo del entero sistema burocrático de las universidades ecuatorianas, con miras a la eliminación de estorbos y a la agilización de los procedimientos. Para ello, y para el desempeño de otras funciones que se enunciarán más adelante, podrían constituirse equipos de 
${ }^{3}$ Lógicamente, la organización práctica formas muy diversas,

por lo que la propues-

ta orientadora que aquí se recoge pretende solo servir como guía. Puesto que las circunstancias varían de un caso a otro, sería contraproducente la adopción de un único modelo referencial.

4"Las políticas y líneas propuestas deben mostrar la planificación en términos de los equipos humanos especializados y/o multidisciplinarios gadores, aststentes de investigacion), los recurso asignados para la investigación, la asistencia a conferencias, los reportes o artículos de investigación (papers), los me canismos de incentivos,

y una vinculación clar con los posgrados que dicta la IES" (CEAACES, trabajo, de carácter consultivo, integrados por docentes de tiempo completo a los que, durante el período en que se desarrolle el proceso, se dispensaría de tareas que no fueran estrictamente docentes y de investigación, con una asignación horaria semanal de clases inferior a diez horas. Esas personas estarían en estrecha comunicación con las direcciones académica y administrativa de sus centros de adscripción, que les suministrarían cuanta información requiriesen. Tras la disolución de esos equipos de trabajo, una vez terminado su cometido, se designaría -por vía electoral- a una de las personas partícipes en el proceso para presidir el Consejo de Investigación (institución de que se tratará más adelante, cuya puesta en funcionamiento se prevé después del término de las labores de los grupos de trabajo).

Entre las competencias de esos equipos figuraría la elaboración de un estudio sobre el diseño -o rediseño- de un Departamento de Investigaciones, que debería gobernarse por un órgano colegiado dependiente directamente del Rectorado. Los integrantes de esos grupos de trabajo asumirían también la acometida de una revisión a fondo de los procedimientos burocráticos vigentes en su universidad.

Esos equipos se ocuparían también del estudio de posibles mecanismos alternativos a los actuales controles biométricos (allá donde se hayan instaurado), de modo que se eliminen las trabas para la realización de trabajos de campo y para la participación en eventos académicos. Naturalmente habrá que tomar en cuenta las imposiciones gubernamentales que rigen para todas las instituciones universitarias del país.

El Consejo de Investigación cuya creación se sugiere 3 será un órgano de carácter consultivo, dependiente directamente del Rectorado, renovable cada cuatro años, conformado por un presidente y dos vocales, cuyo nombramiento compete al Rector de la institución, y dotado de las siguientes competencias:

1) impulsar el proceso de reorganización del Departamento de Investigaciones;

2) configurado como centro de formación de investigadores, alentará e impulsará la constitución de equipos de investigación en las diversas facultades, en conformidad con las exigencias del CEAACES ${ }^{4}$, y promoverá la iniciación en tareas investigadoras de los profesores que vayan incorporándose a la universidad, en cuyo horario semanal habrá de reservarse un hueco obligado para esa actividad; 
3) captar recursos económicos para la investigación, en estrecho contacto con el Departamento de Investigaciones;

4) promover la movilidad y la participación de los docentes en redes de investigación y en congresos, y suscitar alianzas interdisciplinarias para la investigación;

5) gestionar la visualización de las investigaciones de los docentes en la web de la institución y en los blogs que se pongan en marcha.

El presidente del Consejo estará exento de tareas docentes y administrativas, y asumirá la representación de la universidad en el ámbito investigador ante instancias externas, entre las que han de incluirse con carácter preferencial las que conforman el tejido empresarial de la provincia donde radique la sede de la institución. Y dirigirá personalmente un grupo de investigación en la facultad a que esté adscrito.

Para reforzar la imprescindible búsqueda de recursos económicos que permitan convertir en realidad muchas de las aspiraciones enunciadas en esta propuesta habría de promoverse la organización de una Asociación de Antiguos Alumnos en cada universidad.

Deben diseñarse asimismo estrategias que aseguren un espacio privilegiado para la investigación en la universidad y posibiliten que el proceso de reformas que aquí se sugiere sirva para asentar una nueva concepción del quehacer universitario en la que la actividad investigadora deje definitivamente de ser la invitada de piedra. Entre esas estrategias, anticipadas en las páginas que preceden, se recomiendan las siguientes:

1) capacitación permanente en investigación del personal docente, desde cursos y talleres impartidos de modo periódico en torno a métodos y técnicas de investigación y de escritura, hasta estudios de Maestría, Doctorado y Posdoctorado, que, además, permitirán anudar lazos con otras universidades y estimular procesos de movilidad de profesores;

2) apoyo a las actividades de investigación que realiza el docente, con objeto de a) garantizar y proteger la disponibilidad de tiempo; b) habilitar los recursos económicos necesarios para la compra de equipos, para el financiamiento del plan de estímulos a la productividad, y para atender a los gastos de participación en congresos, y c) agilizar las condiciones de movilidad; 
3) constitución en cada universidad de grupos de investigación de carácter multidisciplinar, aglutinados en torno a problemas considerados prioritarios por el Consejo de Investigación y el Departamento de Investigaciones, y organización de departamentos en los que queden integrados esos equipos de trabajo, que habrán de laborar desde una perspectiva interdisciplinar y proponerse la formación de investigadores singulares (no solitarios), capaces de coordinar su esfuerzo con el de otros colegas;

4) elaboración de programas y concursos que sirvan de estímulo a la investigación, tanto para los docentes como para los estudiantes;

5) participación de los alumnos en los proyectos y grupos de investigación mediante la inclusión de los proyectos en el Syllabus de las diversas materias, con el fin de que desarrollen las competencias que la sociedad espera de ellos al término de sus estudios, y para propiciar una interacción teoría-praxis;

6) participación de estudiantes de los últimos cursos en congresos y seminarios;

7) muchísima más exigencia en los procesos de tesis de grado y de maestría, de modo que los asesores se involucren en una orientación efectiva, atenta a los aspectos metodológicos y a la calidad literaria de los textos;

8) facilitación del acceso a becas para estudios de maestría y de doctorado de los alumnos más capacitados, con la perspectiva de un futuro vínculo a la institución;

9) renovación de los planes de investigación de las universidades, que han de diversificarse y desarrollarse en armonía y complementariedad con los que se siguen en otras entidades de educación superior del mismo entorno geográfico.

\section{Recapitulación final}

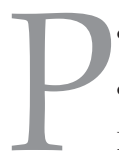

ara cerrar estas consideraciones, indudablemente apresuradas y sujetas a revisión, por cuanto versan sobre una realidad que evoluciona con rapidez, aunque a diferentes ritmos, según regiones geográficas, queremos asumir el propósito formulado por Ayolaida Rodríguez Miranda para el Programa de Capacitación en Investigación para Docentes que propone en el informe al que tantas veces hemos recurrido en estas páginas:

contribuir a la consolidación de una cultura de la investigación científica en las instituciones de educación superior, mediante la formación de los docentes en investigación con una postura crítica ante las bases filosóficas y prácticas de la 
investigación científica, que les permita realizar actividades de investigación para la generación de conocimientos científicos en sus áreas de competencia, desde la perspectiva de una formación integral, ética, científica, pedagógica, humanística y tecnológica (Rodríguez Miranda, A., 2015).

Un exceso de control y una preocupación desmedida por la planificación acaban coartando las potencialidades de los investigadores, que han de llevar a cabo su trabajo con creatividad y desde la persuasión de que vocacionalmente han asumido un compromiso muy serio con la sociedad, que ha puesto en sus manos los instrumentos para impulsar cambios que sustenten un desarrollo que, por definición, se riñe con improvisaciones y con aceleración de los tiempos que requieren los diversos procesos.

\section{BIBLIOGRAFÍA}

CEAACES. (2013 a). Evaluación, acreditación y categorización institucional. Fichas técnicas (http:// www.ceaaces.gob.ec/sitio/wp-content/uploads/2013/10/Fichas-Te\%CC\%81cnicas.pdf)

CEAACES. Dirección de Acreditación de Universidades y Escuelas Politécnicas (2013 b). Informe General sobre la Evaluación, Acreditación y Categorización de las Universidades y Escuelas Politécnicas (http://www.ceaaces.gob.ec/sitio/wp-content/uploads/2014/01/Informe-3.pdf)

Fernández Pérez, R.; Gámiz Ruiz, M. A.; García Martínez, M. A.; Moraga Ávila, J.; Peña Sánchez, J. \& Porras Mínguez, S. (2012). "Calidad educativa y espacios de trabajo universitarios". En ReiDoCrea. Revista Electrónica de Investigación Docencia Creativa. Universidad de Granada. Vol. 1. Art. 17: 126-131 (http://www.ugr.es/ miguelgr/ReiDoCrea-Vol.1-Art.17-Fernandez-GamizGarcia-Moraga-Pena-Porras.pdf)

Pérez Escobar, Jacobo. (2013). Metodología y técnica de la investigación jurídica. Bogotá: Editorial Temis

República del Ecuador. (2013). Plan Nacional de Desarrollo, Plan Nacional para el Buen Vivir 2013-2017. Quito: Secretaría Nacional de Planificación y Desarrollo

Rodríguez Miranda, Ayolaida. (2015). Producción de conocimiento científico y tecnológico universitario en la Zona 1 y su responsabilidad social en el marco del Plan Nacional del Buen Vivir. Ibarra: Secretaría de Educación Superior, Ciencia, Tecnología e Innovación

Roig, Arturo Andrés. (2013). Esquemas para una historia de la filosofía ecuatoriana. Quito: Universidad Andina Simón Bolívar-Corporación Editora Nacional

Ruiz, Juan Carlos. (2001). "La investigación científica en el Ecuador". En Revista Ecuatoriana de Medicina Crítica. Quito. Vol. 2, No. 2 (diciembre) http://www.medicosecuador.com/medicina critica/rev_vol2_num2/la_investigaciona.html

Subsecretaría de Planificación. Zona 1-Norte. (2013). Agenda Zonal 1. Provincias de Esmeraldas, Imbabura, Carchi y Sucumbíos 2013-2017.

Villavicencio, Arturo. (2013). ¿Hacia dónde va el Proyecto Universitario de la Revolución Ciudadana? Quito: 13 ediciones. 